\title{
Zwischen den Stühlen. Das Berliner Journal für Soziologie in der gesellschaftlichen Transformation und Refiguration
}

\author{
Klaus Dörre · Frank Ettrich · Karin Lohr • Martina Löw • \\ Hartmut Rosa $\cdot$ Benjamin Seyd
}

Angenommen: 13. September 2021 / Online publiziert: 5. Oktober 2021

(C) Der/die Autor(en) 2021

\section{Die Soziologie und das Berliner Journal vor neuen Herausforderungen}

Es hätte der gegenwärtigen Corona-Pandemie nicht bedurft, um festzustellen: Wir leben und arbeiten in einer Zeit vielfältiger globaler Krisenprozesse, die interagieren und sich gegenseitig verstärken. Für eine deutschsprachige Fachzeitschrift wie das Berliner Journal für Soziologie stellen sich inmitten gesellschaftlicher Beschleunigungsprozesse, räumlicher Refiguration und multipler Krisendynamiken zudem Probleme und Fragen, die nur auf den ersten Blick marginal erscheinen. Erinnern wir uns an ein Ereignis, das 2019 hohe Wellen schlug und im Bundestagswahlkampf 2021 seinen Widerhall gefunden hat: ,Wir haben noch neun Jahre Zeit, um den Planeten Erde vor der Selbstverbrennung zu retten, und die Politik tut nichts!“, lautete

\footnotetext{
Klaus Dörre $(\bowtie) \cdot$ Hartmut Rosa $\cdot$ Benjamin Seyd

Friedrich-Schiller-Universität Jena, Jena, Deutschland

E-Mail: Klaus.doerre@uni-jena.de

Hartmut Rosa

E-Mail: hartmut.rosa@uni-jena.de

Benjamin Seyd

E-Mail: benjamin.seyd@uni-jena.de

Frank Ettrich

Universität Erfurt, Erfurt, Deutschland

E-Mail: frank.ettrich@uni-erfurt.de

Karin Lohr

Humboldt-Universität zu Berlin, Berlin, Deutschland

E-Mail: karin.lohr@sowi.hu-berlin.de

Martina Löw

Technische Universität Berlin, Berlin, Deutschland

E-Mail: martina.loew@tu-berlin.de
} 
die Botschaft eines Videos, das kurze Zeit nach seiner Veröffentlichung nahezu 15,5 Mio. Menschen angeklickt und mehr als 1,5 Mio. ,geliket" hatten. Binnen weniger Wochen erreichte der Youtuber Rezo ein Publikum, das die jährlichen Downloadzahlen des Berliner Journals für Soziologie um mehr als das 150-fache übertraf.

Das Beispiel wirft Fragen auf - auch für die Soziologie und das Berliner Journal. Weitgehend offen ist, wie sich die Soziologie zu den grundlegenden gesellschaftlichen Problemen einerseits und in einer radikal veränderten Medienlandschaft andererseits positionieren soll und welche Funktion eine soziologische Zeitschrift dabei übernehmen kann. Man könnte sogar fragen: Hat es in einer digitalisierten, beschleunigten Welt überhaupt noch einen Sinn, eine referierte Fachzeitschrift herauszugeben, die sich allein schon wegen des formalisierten Begutachtungsverfahrens und der akribischen Redaktionsarbeit Beschleunigungszwängen entzieht? Erschöpft sich die Aufgabe eines solchen Journals künftig darin, Soziolog_innen zu Qualitätsnachweisen für ihre Forschungen zu verhelfen und wissenschaftliche Karrieren voranzubringen? Oder kann die Soziologie - auch vermittelt über ein fachliches $\mathrm{Pu}-$ blikationsmedium - zum gesellschaftlichen Diskurs über zukünftige Entwicklungspfade beitragen? Kurzum: Benötigt die ,scientific community“ überhaupt noch eine deutschsprachige Fachzeitschrift wie das Berliner Journal für Soziologie?

Wir meinen: eindeutig ja.

Dabei sei zuallererst gesagt: Das Berliner Journal ist eine allgemeine soziologische Fachzeitschrift, die der gesamten Disziplin als Publikationsorgan offensteht. Das wird auch in Zukunft so bleiben. Kein theoretischer Ansatz, keine Methode soll bevorzugt werden oder ausgeschlossen sein. Themenvielfalt und Perspektivenpluralität sind unbedingt erwünscht. Alle Spezialdisziplinen der Soziologie können mit gleichbleibender Aufmerksamkeit seitens der Herausgeber_innen und der Redaktion rechnen. Selbstverständlich werden informative Artikel auch dann publiziert, wenn sie Resonanz hauptsächlich in kleinen Fachkreisen erzeugen. An einem Prinzip möchten wir allerdings festhalten: Was für uns vor allem zählt, ist die Grundidee, die einen Artikel auszeichnet. Überzeugen Idee und methodische sowie textliche Ausführung, steht einer Veröffentlichung prinzipiell nichts im Wege.

Dennoch veranschaulichen das eingangs angeführte Video und die Reaktionen, die es ausgelöst hat, die Herausforderungen, mit denen sich die Soziologie, ihre Publikationsorgane eingeschlossen, heute auseinandersetzen muss. Dazu zählen erstens die im Gange befindliche gesellschaftliche Transformation und Refiguration, zweitens daraus erwachsende methodologische Schwierigkeiten der Soziologie als Disziplin sowie drittens schließlich ein tiefgreifender Strukturwandel der Öffentlichkeit, der an soziologischen Fachzeitschriften nicht vorbeigeht. Alle diese Herausforderungen berühren das Berliner Journal in Form und Inhalt; sie haben Einfluss auf die Programm- und Heftgestaltung. Nachfolgend möchten wir darlegen, welche Anliegen und Aufgaben wir künftig, neben vielem Altbewährten, mit der Zeitschrift verbinden. 


\section{Transformation und Refiguration, revisited}

Der erste Punkt bezieht sich auf die inhaltliche Ausrichtung und ist eng verbunden mit der Geschichte des Journals. Die Gründung der Zeitschrift 1991 ging auf eine Initiative der ostdeutschen Gesellschaft für Soziologie zurück, die sich ein Jahr zuvor als erste selbstständige Fachgesellschaft von DDR-Soziolog_innen konstituiert hatte. Dem Selbstverständnis nach eine allgemeine soziologische Fachzeitschrift, die Beiträge aus allen Forschungs- und Themenbereichen der Soziologie publiziert, war das Berliner Journal von Beginn an in besonderer Weise mit der Systemtransformation, und das hieß zunächst natürlich vor allem mit dem Übergang von staatsbürokratisch-sozialistischen zu marktwirtschaftlich-kapitalistischen Gesellschaften befasst (Ettrich 1991; Zapf 1994). Wie neuere Forschungen (z. B. Mau 2019; Hillebrand 2014; Haag et al. 2017) belegen, ist dieser Umbruch keineswegs abgeschlossen. Teile der ostdeutschen Subgesellschaft verstehen sich noch immer als sozial, kulturell und politisch erheblich vom Westen unterschieden und werden als solche auch medial und wissenschaftlich beschrieben. Andere Gruppen haben hybride ost-westund west-ost-deutsche Praktiken ausgebildet, die Gesellschaft innovieren. Auch die Prognose, wonach die Implosion des Staatssozialismus den Prozess der europäischen Integration erheblich beeinflussen und die politische Institutionenordnung Europas verändern werde, hat sich bewahrheitet (Ther 2014), weshalb auch dieses Thema relevant bleibt.

Wenn jedoch heute von Transformation gesprochen und geschrieben wird, sind in der Regel andere Dynamiken gemeint. Schon während des Systemumbruchs hatte sich abgezeichnet, dass der soziale Wandel von Krisensymptomen und gesellschaftlichen Spannungen überlagert wird, die nicht dem Staatssozialismus zuzurechnen sind. Hier ist zum einen auf Funktionsmechanismen, Verwerfungen und Konfliktlinien westlicher Kapitalismen zu verweisen, denn spätestens seit der globalen Finanzund Wirtschaftskrise am Ende der Nullerjahre bestimmen diese Treiber Themen und Richtung des sozialen Wandels. Zum anderen beobachten wir weltweit, also nicht nur im Westen, Prozesse der Refiguration durch global gestiegene Vernetzungen und Abhängigkeiten, wachsende Mobilität und schließlich auch Digitalisierung. Vermeintlich getrennte Strukturebenen, Scales oder Konstruktionen von Virtualität und Realität werden als miteinander verknüpft und verwoben erfahren. Neben der zeitlichen Beschleunigung sozialer Veränderungsprozesse kommt es zu einer Refiguration von Räumen; in der Folge weicht die in der Moderne dominante Raumvorstellung (Container) auf - man kann stattdessen von einem Zusammenspiel und Kräfteverhältnis von mehreren gleichzeitig wirksamen Raumfiguren sprechen (Territorial-, Netzwerk-, Bahnenräume, Orte). Die Folge sind sich polarisierende Raumlogiken, widersprüchliche und polykontexturale Handlungsanforderungen sowie ein Wandel im Raumwissen (Löw und Knoblauch 2021).

Auch deshalb sehen sich - hierin besteht Übereinstimmung weit über die Grenzen der Soziologie hinaus - die früh industrialisierten Länder auf besondere Weise mit einem historisch neuartigen Veränderungsdruck konfrontiert (Dörre et al. 2018). Sie befinden sich möglicherweise am Beginn eines gesellschaftlichen Wandels, der in seinen Verläufen, Be- und Verarbeitungsformen zwar nicht unbedingt identisch, wohl aber in seiner Bedeutung mit jener „Great Transformation“ vergleichbar ist, die 
der Wirtschaftshistoriker Karl Polanyi Mitte des 20. Jahrhunderts so eindrucksvoll beschrieben hat (Polanyi 1995 [1944]). Stärker als zu Polanyis Zeiten geht der Veränderungsdruck heute allerdings von der ökologischen Konfliktachse aus. Daher ist es kein Zufall, wenn Naturwissenschaftler die Unausweichlichkeit eines radikalen Wandels besonders klar aussprechen, denn:

Es eilt sehr. Ein Systemkollaps ist eine reale Gefahr [...]. Wir stehen vor gewaltigen Herausforderungen bedingt durch das rasante Bevölkerungswachstum, die Übernutzung der Ressourcen, die Veränderung des Klimas, den Verlust der Biodiversität, und insgesamt erleben wir einen schleichenden Verlust der Lebensgrundlagen. (Weizsäcker 2020, S. 82)

Dieser Umbruch ist außerordentlich vielschichtig. Er wirft die Frage nach dem Verhältnis von Ökologie, Ökonomie und Sozialem auf, er umfasst eine Vielzahl an Dimensionen, betrifft alle Ebenen und Teilsysteme der Gesellschaft, er berührt die gesellschaftliche Kultur und die individuelle Lebensführung. Er tangiert daher unvermeidlich auch das Selbstverständnis der Soziologie in ihrer Gesamtheit. Mit dieser Feststellung sind die Gemeinsamkeiten innerhalb des Faches aber fast schon aufgebraucht.

Die Liste an divergenten Deutungsversuchen der im Gange befindlichen gesellschaftlichen Umbruchprozesse, ihrer Triebkräfte, Spielräume und Zielhorizonte ist groß. Anstelle eines wohlklingenden Orchesters ertönt ein dissonanter Chor. Dass die Soziologie über Ursachen, Dynamiken, Koordinaten und Reichweite gesellschaftlichen Wandels streitet, ist indes wenig erstaunlich, vielmehr geradezu erwünscht und deshalb eigentlich kein Problem. Um im besten Wortsinn aufklärerisch wirken zu können, benötigt das Fach keinen einheitlichen Forschungsstand zur Transformation und Refiguration. Angebracht ist ein Theorie-, Analyse- und Methodenpluralismus, der die Vielfalt des Wandels in ausdifferenzierten Gesellschaften mit Hilfe gemeinsamer Anstrengungen angemessen abbildet. Pluralismus darf jedoch nicht mit Beliebigkeit verwechselt werden. Wichtig ist die - in ihrer Form auf Verständigung angelegte - konstruktive Kontroverse, die neben allen Divergenzen auch nach dem Gemeinsamen sucht. Es sind vor allem drei Themenbereiche, die aus unserer Sicht förmlich nach soziologischer Expertise und Debatte schreien.

Ein erster Themenbereich ergibt sich aus der Mehrdimensionalität der im Gange befindlichen Transformation und Refiguration. Vor dem Ausbruch der CoronaPandemie schien es, als rage unter den globalen Herausforderungen der anthropogene Klimawandel wegen der Dringlichkeit seiner Bearbeitung besonders heraus. Die Welt steuert gegenwärtig eher auf eine Erdaufheizung um $3 \mathrm{Grad}$ als auf das vom Weltklimarat (IPCC) avisierte 1,5-Grad-Ziel zu. Dies mit der Folge, dass große Teile der Erde unbewohnbar werden könnten (IPCC 2018; Rogelj et al. 2019; UNEP 2019). An der Dramatik entsprechender Szenarien hat sich trotz des vorübergehenden Rückgangs klimaschädlicher Emissionen, den die Corona-Krise mit sich gebracht hat, nichts geändert. Dennoch haben die Pandemie und die tiefe Rezession den Klimawandel zwischenzeitlich von seinem Spitzenplatz auf den politischen Agenden verdrängt. Darin offenbart sich eine Grundproblematik der neuen Transformation. Wer den ökologischen Gesellschaftskonflikt für die zentrale Triebkraft sozialen Wandels hält, wird bei dessen Bearbeitung auf zahlreiche andere soziale 
Felder stoßen, die mit den Gesellschaft-Natur-Beziehungen verwoben sind, jedoch nach eigenen Regeln und je spezifischen Strukturen funktionieren. Von besonderem Interesse ist dabei derzeit etwa die Frage, ob die Prozesse einer immer engeren transnationalen Verflechtung bzw. einer ,,intensivierten Globalisierung“ (Giddens 1995) weiter anhalten, ob sie ins Stocken geraten oder sich sogar umkehren (Menzel 2021) und welche Rolle die Digitalisierung hierbei spielt.

Mehrdimensionalität und Polykontexturalität der Entwicklung schließen jedenfalls aus, dass der ökologische Gesellschaftskonflikt andere soziale Fragen einfach verdrängt. Im Gegenteil: Akteure, die sich vornehmlich auf der ökologischen Konfliktachse verorten, müssen, häufig in Konkurrenz zu den Akteuren in anderen sozialen Feldern, um Aufmerksamkeit und Durchsetzungsfähigkeit ringen. Ob und wie die verschiedenen Veränderungsdynamiken in Gesellschaften mit sehr ungleichen Machtbalancen aufeinander einwirken, ist die eigentlich spannende Frage, die es mit soziologischen Mitteln zu beantworten gilt.

Daraus ergibt sich ein zweiter Themenbereich, der aus der tendenziellen Macht-, Herrschafts- und Konfliktvergessenheit rein ökologischer Aufklärung resultiert. Die Ausrufung des neuen Erdzeitalters Anthropozän stellt klar, dass sich die Gesellschafts-Natur-Beziehungen fundamental verändert haben. Im ,web of life“ reproduziert sich Natur immer mehr über moderne Gesellschaften, und moderne Gesellschaften reproduzieren sich durch die von ihnen erzeugten Naturverhältnisse (,,double internality“, Moore 2015, S. 1). Die Rede vom Menschenzeitalter darf jedoch nicht den Blick dafür verstellen, dass die Menschheit eben nicht als aufgeklärtes Kollektiv handelt. Agiert wird in ausdifferenzierten, vermachteten, arbeitsteilig organisierten, vergeschlechtlichten, sozial gespaltenen und politisch häufig polarisierten (kapitalistischen) Gesellschaften. Anders als Ulrich Beck (1986) es erwartete, ist die Logik der ökologischen Risikoverteilung unauflöslich mit der Logik der Reichtumsverteilung, aber auch mit den Konflikten um „race“ und „gender“ verbunden (Federici 2015; Arruzza et al. 2019). Wie diese Konfliktlinien aufeinander einwirken, in welcher Weise Klassenspannungen, ökologischer Gesellschaftskonflikt und Auseinandersetzungen um sexistische oder rassistische Dominanz interagieren, lässt sich nur mittels empirischer Forschungen klären. Naheliegend ist jedoch, dass der Großkonflikt um eine ökologisch wie sozial nachhaltige gesellschaftliche Ordnung zwischen unterschiedlichen Lagern mit stark divergierenden Interessen und konkurrierenden politischen Ausrichtungen geführt wird. Soziolog_innen verfügen hier durchaus über ein Spezialwissen (Dahrendorf 1957) - und dieses Wissen gilt es zu erneuern, zu erweitern und fruchtbar zu machen. Dabei dürfte das Verhältnis von Wachstum, Nachhaltigkeit und Demokratie zu den Schlüsselproblemen gehören, die es genauer zu erforschen gilt.

Solche Anliegen führen zu einem dritten Grund, der soziologische Expertise regelrecht einklagt - die Möglichkeit und das Problem einer neuerlichen Systemtransformation. Vor dem Hintergrund der beschriebenen Entwicklungen und Herausforderungen stellt sich das Problem politischer Ordnungsbildung auf neue Weise. Es liegt nahe, dass sich künftig jede Politik zu Fragen ökologischer und sozialer Nachhaltigkeit verhalten muss (Adloff und Neckel 2019, S. 167). Eine weitgehend offene Frage politischer Soziologie ist jedoch, wie Produktions- und Lebensweisen künftig 
zu organisieren sind und wie sich Ökologie, Ökonomie und Soziales im Sinne eines globalen demokratischen Zusammenlebens von Menschen zusammendenken lassen.

Gleich, wie man sich im Spektrum politischer Utopien positioniert, gilt dabei: An den Grenzen naturwissenschaftlicher Expertise offenbaren sich Chancen und Verpflichtungen der zeitgenössischen Sozialwissenschaften. Selbstverständlich kann und darf sich die Soziologie als Fach nicht auf ein favorisiertes Gesellschaftsmodell einigen. Aber heißt dies, dass sie sich einer Diskussion über den Typ von Gesellschaft, der die Probleme ökologischer und sozialer Nachhaltigkeit am besten zu bewältigen vermag, zu verweigern hat?

Wie man es auch dreht und wendet - eine Wissenschaft von der Gesellschaft darf den großen Zukunftsproblemen nicht ausweichen. Wir halten es deshalb für wichtig, die Debatte um sozialen Wandel weiter zu führen und überdies dabei auch den nationalen Diskursraum zu verlassen. Mit diesem Vorhaben beginnen wir keineswegs bei Null. Neben dem ersten Sonderband des Berliner Journals (Dörre et al. 2019) haben die Beiträge des Doppelhefts zu „Wachstum und Demokratie“ (Heft 1-2/18), unsere Corona-Kontroverse (Seyd 2020; Dörre 2020; Rosa 2020; Lessenich 2020b) sowie diverse Einzelbeiträge (u.a. Mau et al. 2020; Lessenich 2020a; Dolata 2019; Brand 2018) den Transformationsfaden in der Zeitschrift längst wieder aufgenommen. Künftig wollen wir dafür sorgen, dass die Debatte mit größerer Kontinuität und Systematik geführt wird. Diese Diskussion bedarf ,,soziologischer Imagination“ (Wainwright und Mann 2018; Norgaard 2017) und des Mutes von Soziolog_innen, sich gegebenenfalls zwischen alle Stühle zu setzen. Weder darf es darum gehen, den Einflussreichen und Mächtigen nach dem Munde zu reden, noch ist vordergründige Kumpanei mit sozialen Bewegungen oder anderen oppositionellen Kräften angebracht. Es zählt auch hier die Idee, die Bereitschaft und Fähigkeit, über das Bekannte hinauszugehen, wo es der Prüfung und gegebenenfalls der Veränderung bedarf.

\section{Die methodologische Herausforderung der Soziologie}

Mit diesem Programm stoßen wir zweifellos auf ein methodologisches Problem, nicht zuletzt mit Blick auf die altbekannte Frage nach der Werturteilsfreiheit. Nachhaltigkeitsziele, wie sie in den 17 Sustainable Development Goals festgeschrieben $\operatorname{sind}^{1}$, stellen weit mehr dar als eine unverbindliche Auflistung unbewältigter Probleme. Nachhaltigkeit wird von ,nationalen Gesellschaften, (trans)nationalen Organisationen, Städten, Unternehmen und sozialen Bewegungen als normatives Prinzip

\footnotetext{
${ }^{1}$ In Kurzfassung lauten die 17 SDGs: 1. Armut beenden; 2. Ernährung sichern; 3. Gesundes Leben für alle; 4. Bildung für alle; 5. Gleichstellung der Geschlechter; 6. Wasser und Sanitärversorgung für alle; 7. Nachhaltige und moderne Energie für alle; 8. Nachhaltiges Wirtschaftswachstum und menschenwürdige Arbeit für alle; 9. Widerstandsfähige Infrastruktur und nachhaltige Industrialisierung; 10. Ungleichheit verringern; 11. Nachhaltige Städte und Siedlungen; 12. Nachhaltige Konsum- und Produktionsweisen; 13. Sofortmaßnahmen ergreifen, um den Klimawandel und seine Auswirkungen zu bekämpfen; 14. Bewahrung und nachhaltige Nutzung der Ozeane, Meere und Meeresressourcen; 15. Landökosysteme schützen; 16. Frieden, Gerechtigkeit und starke Institutionen; 17. Umsetzungsmittel und globale Partnerschaft stärken (BMZ 2020).
} 
proklamiert“ (Adloff und Neckel 2019, S. 167). Diese normative Setzung ist im Begriff des Anthropozäns selbst angelegt. Die Begriffsschöpfung des Nobelpreisträgers Paul J. Crutzen (2019) ist empirisch begründet, doch der Begriff selbst drängt nach „Hypothesen über das, was kommen könnte“ (Gerhardt 2019, S. 84). Die darin angelegte Verbindung von empirischer Feststellung und globaler Erwartung ist, so der der Ethiker Volker Gerhardt, auch ,aus wissenschaftstheoretischer Sicht von größter Bedeutung“" (ebd., S. 86).

Was bedeutet diese Feststellung für eine Soziologie, die sich in der Tradition Max Webers dem Grundsatz der Werturteilsfreiheit verpflichtet fühlt? Wir können hier keinen Widerspruch erkennen. Webers Postulat der Werturteilsfreiheit ist auch für empirische Wissenschaften mit der Vorarbeit einer Wertediskussion untrennbar verknüpft, wie Webers Euvre hinlänglich belegt (s. dazu a. BJS Heft 1/2021). Was die Soziologie für die Gesellschaft in diesem Zusammenhang leisten kann, ist nicht die politische Leistung einer klaren Handlungsempfehlung, sondern eine solche Wertediskussion im Sinne Webers. Deren Kern besteht darin, den handelnden Akteuren ihre Optionen, die Kosten und Folgen ihrer Optionen sowie deren doppelte Wertbezogenheit vor Augen zu halten. Im Falle ihres Gelingens liegt ein Stück ,,soziologischer Aufklärung“ vor: „Wenn Ihr Nachhaltigkeit zum obersten Leitwert machen wollt, dann sind diese Handlungsweisen und institutionellen Arrangements erfolgversprechend, jene aber nicht.“ Oder: „Wenn die Menschenrechte (oder die SDGs, oder die Demokratie) für Euch Verpflichtungscharakter haben, dann ist diese Politik konsequent, jene aber nicht.“ Das ist die eine Seite einer solchen Wertbeziehung, die Weber mit der Metapher der kämpfenden Götter umschreibt. Die andere Seite besteht darin, den Akteuren vor Augen zu führen, welchen „Göttern“ sie in letzter Konsequenz dienen, wenn sie einen gegebenen Handlungskurs fortsetzen oder ein institutionelles Regime aufrechterhalten - und sie auf diese Weise dazu anzuregen, ggf. besser einen anderen Pfad einzuschlagen.

$\mathrm{Zu}$ den methodologischen Grundfragen, die sich angesichts der ,geistesgeschichtlichen Lage“ der Soziologie heute stellen, gehört aber noch weit mehr als der Streit über ihre Wertfreiheit und Wertbezogenheit. Die in jüngster Zeit wieder intensiv geführte Debatte darüber, was als soziologisches Wissen auftreten darf und wie dieses Wissen zu produzieren sei, in welchem Verhältnis also qualitative, quantitative und interpretative Ansätze zueinander stehen sollen, lässt sich gewiss nicht ein für allemal entscheiden; sie muss immer wieder neu geführt werden. Dazu gehört die Auseinandersetzung darüber, welche Soziologie welche Art von Theorie benötigt und inwiefern und auf welche Weise die Soziologie auch eine Wissenschaft der Raum- und Zeitverhältnisse sein muss. Auch dafür will das Berliner Journal ein Ort sein. Anders als manche andere Zeitschrift folgen wir jedoch der Gewissheit, dass sich die Soziologie nicht quasi-szientifisch auf eine ,harte“, erklärende Wissenschaft reduzieren lässt und dass sie auch eine deutende Kulturwissenschaft sein muss und sein kann, wenn sie gesellschaftlich relevant bleiben will. 


\section{Das Berliner Journal im Strukturwandel der Öffentlichkeit}

Die Soziologie wird in der gesellschaftlichen Transformation nur dann gebraucht, wenn sie Wissen erzeugt, das auf andere Weise nicht entstehen kann. Wissen meint hier zunächst in der humanistischen Tradition eine wahre und/oder wohlbegründete Meinung. Dies schließt ein, dass es, wie etwa die Leugnung des anthropogenen Klimawandels illustriert, Meinungen geben kann, die weder wohlbegründet noch wahr sind. In unserem Fall muss die Wahrheitssuche darauf ausgerichtet sein, die gesellschaftlichen Entwicklungen angemessen zu beschreiben, analytisch zu durchdringen und die in ihnen angelegten Handlungsspielräume auszuloten. Mit wissenschaftlichen Mitteln produziert, finden die so gewonnenen Erkenntnisse in der gesellschaftlichen Öffentlichkeit nicht automatisch Gehör. Der Strukturwandel gesellschaftlicher Öffentlichkeit(en) bringt es mit sich, dass Soziolog_innen nicht nur mit anderen Wissenschaftsdisziplinen, sondern auch mit Journalist_innen, Sachbuchautor_innen, schreibenden Politiker_innen, Think Tanks und Influencer_innen um Aufmerksamkeit konkurrieren. Darin ist eine Gefahr angelegt, vor der Ralf Dahrendorf noch ohne Kenntnis des Internets eindringlich gewarnt hat. Im Vorwort zu seinem Klassiker Soziale Klassen und Klassenkonflikt in der industriellen Gesellschaft schreibt er, die Öffentlichkeit agiere wie ein ,,verärgerter Gläubiger, der der vorerst zahlungsunfähigen Soziologie ständig im Nacken“ sitze, „um zu verhüten, daß auch nur ein Pfennig ihm aus den Fingern geht“. „Ist es ein Wunder“, fragt der liberale Soziologe rhetorisch, „daß unter diesen Umständen mancher Soziologe dazu übergegangen ist, Falschgeld zu produzieren?“ (Dahrendorf 1957, S. IIV)

Bei aller Berechtigung des Strebens nach öffentlicher Resonanz muss die Soziologie, wie jede andere Wissenschaft auch, Falschgeldproduktion vermeiden. Das ändert aber nichts daran, dass sie sich der Digitalisierung und dem mit ihr verbundenen neuerlichen Strukturwandel der Öffentlichkeit stellen muss. Das BJS ist von den Veränderungen unmittelbar betroffen. Seit Anfang 2020 ist die Zeitschrift dank DEAL-Vereinbarung zu einem Open-Access-Journal geworden. Fast alle Beiträge, die veröffentlicht werden, sind nun für jede und jeden frei zugänglich. Das gedruckte Exemplar gibt es nach wie vor, doch sein Stellenwert hat sich verändert. Diesbezüglich besitzt das BJS wohl einen Vorteil gegenüber allen Zeitschriften, die erst dabei sind, den Sprung in die digitale Welt zu vollziehen.

Gleichwohl bedeutet der Status einer zuvorderst digital verfügbaren Zeitschrift zweierlei. Erstens benötigen wir künftig zusätzliche Aufmerksamkeitsarbeit, um dafür zu sorgen, dass die frei zugänglichen Artikel in den Weiten des Internets auch zur Kenntnis genommen werden. Damit ist zweitens die Öffnung für eine Leserschaft verbunden, die gelegentlich weit über das Fachpublikum hinausgeht. Darin steckt vor allem eine Chance, die aber nur genutzt werden kann, wenn Abstriche an der wissenschaftlichen Fundierung des Publizierten unterbleiben. In diesem Zusammenhang gilt noch immer, was Dahrendorf seiner Zunft ins Stammbuch schrieb:

Wenn aber eine schlecht beratene Öffentlichkeit den Weg der Wissenschaft nicht versteht und mehr verlangt, dann darf und muß der Soziologe den Stolz und das Selbstbewußtsein aufbringen, seine wissenschaftliche Verantwortung gegenüber einer mißverstandenen Verpflichtung zur Gesellschaft, die nur allzu 
oft vom Wunsch zu gefallen beraten ist, zu verteidigen. Hic Rhodus, hic salta! (Dahrendorf 1957, S. IIV)

Dort zu springen, wo Rhodos liegt, heißt aber auch, die Chance zu nutzen, die Grenzen versäulter, gegeneinander abgeschotteter Fachdisziplinen zum Nutzen aller zu überwinden. Zumindest in ihrer ökologischen Dimension stützt sich die aktuelle Transformationsdiskussion in hohem Maße auf naturwissenschaftliches Wissen. Gleiches gilt für die Interpretation der Pandemie, die von Deutungsangeboten aus dem Feld der Medizin dominiert wird. Normwerte, ökologische Belastungsgrenzen und Kipp-Punkte sind jedoch, darauf hatte bereits Ulrich Beck hingewiesen, hochgradig wissens- und definitionsabhängig. Sie sind Gegenstand von Definitionskämpfen im Wissenschaftssystem und in der Gesellschaft. Soziolog_innen können solche Definitionskämpfe mit ihrem Wissen nur bedingt beeinflussen. Sie können aber darauf hinwirken, dass wissenschaftliche Selbstzweifel gesellschaftlich institutionalisiert werden. Sie vermögen, dort anzuknüpfen, wo naturwissenschaftlicher Sachverstand endet. Vor allem können sie jedoch den systematischen, disziplinenübergreifenden Austausch mit Naturwissenschaftler_innen beginnen, die sich zusehends mit den gesellschaftlichen Konsequenzen ihrer empirischen Forschungen beschäftigen. Gelänge ein solcher Brückenschlag (wie er mit den 165.000 Wissenschaftler_innen von „Scientists for Future“ bereits ansatzweise zu erkennen ist), liefe das letztendlich auf einen wissenschaftlichen Paradigmenwechsel hinaus, wie er schon lange gefordert, aber noch höchst selten praktiziert wird.

Ein Paradigmenwechsel, der Sozial- und Naturwissenschaften zu einem produktiven Dialog verhilft, ist ebenso ungewiss wie der Ausgang gesellschaftlicher Transformation und Refiguration. Wir möchten das BJS jedoch zu einem Forum machen, in dem die Debatte um sozialen Wandel sachlich, kontrovers und disziplinenübergreifend geführt wird. Dabei können Sie uns helfen. Füttern Sie uns - und etwa auch unsere neue Rubrik „Debatte“ - mit Ihren Beiträgen! Wir werden unsererseits potenzielle Autor_innen um Beiträge bitten, die geeignet sind, die Diskussion voranzutreiben. In Ausnahmefällen können das, soweit es sich um Muttersprachler_innen handelt, auch englischsprachige Artikel sein. Im Grundsatz bleibt das BJS aber eine deutschsprachige Zeitschrift.

Auf die neuen Heft-Ausgaben des BJS macht seit kurzem ein neu gestalteter Newsletter aufmerksam ${ }^{2}$, zudem informiert unser Twitter-Account ${ }^{3}$ laufend über Neuerscheinungen und sonstige redaktionelle Neuigkeiten. Die Redaktion des BJS ist für die nächsten Jahre in Jena, Erfurt und Berlin angesiedelt. Zum Redaktionsteam gehören Benjamin Seyd, Andreas Häckermann und Henri Band. Ein Netzwerk von Gutachter_innen sorgt dafür, dass eingereichte Manuskripte rasch und sorgfältig bearbeitet werden. Ein unter Federführung von Hans-Peter Müller neu zusammengesetzter Beirat wird die Arbeit von Redaktion und Herausgeber_innen begleiten. Auch der Herausgeber_innen-Kreis der Zeitschrift hat sich personell verändert: Seit Januar 2021 ist Martina Löw und damit auch die Technische Universität Berlin mit an Bord.

\footnotetext{
2 Anmeldung unter https://tinyurl.com/NewsletterBJS.

3 https://twitter.com/BerlinerJournal.
} 
Das alles ist vielleicht wenig spektakulär. Dennoch hoffen wir, dem Veränderungsdruck auf diese Weise trotz begrenzter personeller und materieller Ressourcen Rechnung tragen zu können. Transformation und Refiguration erweisen sich als Abfolge von Bewährungsproben, in denen sich zeigen wird, welche Akteure, Organisationen und Institutionen den Nachhaltigkeitstest bestehen werden. Bei der Beobachtung und Deutung dieser Veränderungen zwischen den Stühlen zu sitzen, ist sicherlich unbequem. In einer Zeit, in welcher viele sich „,nur als verwirrt“ fühlen, weil ,der Boden wankt“, ohne zu wissen, ,warum und von was“ (Bloch 1973 [1959], S. 1), ist das jedoch nicht unbedingt die schlechteste Position.

\section{Das vorliegende Heft}

Das programmatische Interesse, die thematische wie methodische Offenheit und vor allem die Lust an der gesellschaftlichen Selbstbeobachtung spiegeln sich auch in der vorliegenden Ausgabe des BJS. Dass der Streit über gesellschaftliche Zukünfte dabei nicht nur mit Leidenschaft geführt wird, sondern nicht selten auch mit der rhetorischen Herabwürdigung der jeweiligen Kontrahenten einhergeht, ist eine offensichtliche, aber soziologisch doch unausgeschöpfte Beobachtung. Sonja Engel und Dominik Schrage spüren in ihrer kultursoziologischen Studie zum „Spießerverdikt“ einem solchen kommunikativen Muster der Herabsetzung und dessen ordnungsstrukturierendem Einsatz nach. Seit der Romantik kehrt der Vorwurf konformistischer - „spießiger“ - Engstirnigkeit in stets neuen Formen immer wieder. Gleich, ob als Philistersatire in der Romantik, als Kleinbürgerkritik in der Marx'schen Klassentheorie oder in der Selbstinszenierung der Bohemiens: Immer dient der Vorwurf der Spießigkeit in aufgewühlten Zeiten dazu, die Repräsentanten der bestehenden Ordnung zu diskreditieren und gleichzeitig einen alternativen Horizont sozialer Ordnung abzustecken.

Um die Formation nicht sozialer, sondern soziologischer Ordnung geht es bei Elena Beregow: Sie weist der soziologischen Theorieproduktion nach, dass auch diese nicht einfach vom Austausch sachlicher Argumente, sondern - ganz wie gesellschaftliche Großkonflikte auch - von spezifischen „Atmosphären“ zehrt. Dazu tragen nicht zuletzt Kleidungs-, Sprech- und Denkstile bei, die wiederum einer eigenen Theoretisierung bedürfen: Beregow fasst die Theorieatmosphäre als „Produkt des performativen Zusammenwirkens von Text, Lesepraktiken, Dingen und Räumen“. Warum man eher „Luhmannianer“ oder „Deleuzianer“ ist oder gerade nicht, und warum die eine Theorie ,cooler“, die andere dagegen „heißer“ ist, versteht man nach der Lektüre womöglich etwas besser. Und nebenbei wird deutlich, welche Hürden der Werturteilsfreiheit noch entgegenstehen, wenn sich, wie Beregow zeigt, die passende Theorie am Ende vor allem richtig anfühlen muss.

Steht einem der Sinn eher nach Hand- statt nach Kopfarbeit, oder regt sich in einem angesichts theoretischer Komplexitäten gar eine gewisse Sehnsucht nach dem einfachen Leben, haben wir auch dafür etwas im Angebot: die Mixed-MethodsStudie von Anja Eder, Sabine A. Haring-Mosbacher und Franz Höllinger zu Intergenerationenbeziehungen auf österreichischen Bergbauernhöfen, wo wider Erwarten der Drei-Generationen-Haushalt noch immer die Regel darstellt. Aber Obacht: Zwar 
lassen sich in den steiermärkischen Bergen durchaus Anhaltspunkte für einen gelingenden Generationenvertrag, z.T. sogar unter Bedingungen nachhaltigen Wirtschaftens, finden, aber die Alt- und Jungbäuerinnen und -bauern sehen sich auch jeder Menge Herausforderungen gegenüber. Die traditionelle Lebensart zu erhalten, erfordert, wie die Autor_innen zeigen, beinahe immer entschlossene Modernisierung, die ihren Ausdruck im Wandel von der verpflichtenden zur freiwilligen Generationensolidarität, von traditionellen Abhängigkeitsverhältnissen zu einer autonomeren Lebensführung und vom patriarchalen Gehorsam zur egalitären Familienkommunikation findet. Gleichzeitig gehen diese Wandlungsprozesse allerorten mit spezifischen, z.T. wohlbekannten Ambivalenzen einher: Auch in den Bergen sind die Mühen der (modernen) Ebene immer nur einen Katzensprung weit entfernt.

Davon unbenommen sind die Hotspots sozialer Konflikte in den Städten zu finden und wird das Label „Problemviertel“" wohl bis auf Weiteres dem urbanen Raum vorbehalten bleiben. Dass es sich dabei gleichwohl nicht um eine neutrale Beschreibung, sondern vielmehr um eine Konstruktion handelt, die insbesondere durch ,,polizeilich-publizistische Verstärkerkreisläufe“ hervorgebracht wird, zeigen Moritz Rinn und Jan Wehrheim in ihrer ethnographischen Studie zum Essener Stadtteil Altendorf. Die Autoren sensibilisieren für die medialen Mechanismen gesellschaftlicher Problemdefinition: Vor dem Hintergrund verinnerlichter ,territorialer Stigmatisierung“ werden so herunterfallende Eisenplatten als Schüsse gedeutet, die einen zwar unbegründeten, aber nicht unbegründbaren Polizeieinsatz auslösen - denn im „Problemviertel“" gilt es, ,Stärke zu zeigen“.

Derartige symbolische, aber letztlich wenig zielführende Demonstrationen von Handlungsbereitschaft kann man ebenso an anderer Stelle entdecken: nicht zuletzt im Kampf gegen die ökologische Zerstörung unseres Planeten, gegen die - siehe oben - zwar vieles unternommen wird, gegen die aber doch kaum ein Kraut gewachsen scheint. Ingolfür Blühdorn hat das in die Formel von der ,nachhaltigen NichtNachhaltigkeit" gefasst, wonach Nachhaltigkeit zwar stellenweise ernsthaft angestrebt, im Ergebnis aber nie mehr als simuliert wird (u.a. Blühdorn 2018). Aber ist die Hoffnung auf Nachhaltigkeit wirklich nur gewieften Techniken der „Selbstillusionierung“ geschuldet? In seiner Auseinandersetzung mit Blühdorns Ansatz kommt Karl-Werner Brand zu einem klaren Urteil: Blühdorns Annahmen seien historisch wie theoretisch nicht zu halten und führten nicht weiter.

Den Abschluss des Heftes bildet ein sehr persönlicher Nachruf von Michael Schmid auf seinen Freund Bernhard Giesen, der im Dezember 2020 leider verstorben ist.

Funding Open Access funding enabled and organized by Projekt DEAL.

Open Access Dieser Artikel wird unter der Creative Commons Namensnennung 4.0 International Lizenz veröffentlicht, welche die Nutzung, Vervielfältigung, Bearbeitung, Verbreitung und Wiedergabe in jeglichem Medium und Format erlaubt, sofern Sie den/die ursprünglichen Autor(en) und die Quelle ordnungsgemäß nennen, einen Link zur Creative Commons Lizenz beifügen und angeben, ob Änderungen vorgenommen wurden.

Die in diesem Artikel enthaltenen Bilder und sonstiges Drittmaterial unterliegen ebenfalls der genannten Creative Commons Lizenz, sofern sich aus der Abbildungslegende nichts anderes ergibt. Sofern das betreffende Material nicht unter der genannten Creative Commons Lizenz steht und die betreffende Handlung 
nicht nach gesetzlichen Vorschriften erlaubt ist, ist für die oben aufgeführten Weiterverwendungen des Materials die Einwilligung des jeweiligen Rechteinhabers einzuholen.

Weitere Details zur Lizenz entnehmen Sie bitte der Lizenzinformation auf http://creativecommons.org/ licenses/by/4.0/deed.de.

\section{Literatur}

Adloff, F., \& Neckel, S. (2019). Modernisierung, Transformation oder Kontrolle? Die Zukünfte der Nachhaltigkeit. In K. Dörre, H. Rosa, K. Becker, S. Bose \& B. Seyd (Hrsg.), Große Transformation? Zur Zukunft moderner Gesellschaften. Sonderband des Berliner Journals für Soziologie (S. 167-180). Wiesbaden: Springer VS.

Arruzza, C., Bhattacharya, T., \& Fraser, N. (2019). Feminism for the 99\%. A Manifesto. New York: Verso. Beck, U. (1986). Risikogesellschaft. Auf dem Weg in eine andere Moderne. Frankfurt a.M.: Suhrkamp.

Bloch, E. (1973) [1959]. Das Prinzip Hoffnung. Erster Band. Frankfurt a.M.: Suhrkamp.

Blühdorn, I. (2018). Nicht-Nachhaltigkeit auf der Suche nach einer politischen Form. Konturen der demokratischen Postwachstumsgesellschaft. Berliner Journal für Soziologie, 28, 151-180.

BMZ (2020). Agenda 2030: Wo steht die Welt? 5 Jahre SDGs - eine Zwischenbilanz. URL: https://www. 2030agenda.de/sites/default/files/2030/zwischenbilanz/Agenda_2030_Zwischenbilanz_online-2. pdf. Zugegriffen: Juni 2021.

Brand, K.-W. (2018). Disruptive Transformationen. Gesellschaftliche Umbrüche und sozial-ökologische Transformationsdynamiken kapitalistischer Industriegesellschaften - ein zyklisch-struktureller Erklärungsansatz. Berliner Journal für Soziologie, 28, 479-509.

Crutzen, P. J. (2019). Das Anthropozän. Schlüsseltexte des Nobelpreisträgers für das neue Erdzeitalter. München: oekom.

Dahrendorf, R. (1957). Soziale Klassen und Klassenkonflikt in der industriellen Gesellschaft. Stuttgart: Enke.

Dolata, U. (2019). Plattform-Regulierung. Koordination von Märkten und Kuratierung von Sozialität im Internet. Berliner Journal für Soziologie, 29, 179-206.

Dörre, K. (2020). Die Corona-Pandemie - eine Katastrophe mit Sprengkraft. Berliner Journal für Soziologie, 30, 165-190.

Dörre, K., Rosa, H., Becker, K., Bose, S., \& Seyd, B. (Hrsg.). (2019). Große Transformation? Zur Zukunft moderner Gesellschaften. Sonderband des Berliner Journals für Soziologie. Wiesbaden: Springer VS.

Dörre, K., Rosa, H., \& DGS-Vorstand (2018). Great Transformation: Die Zukunft moderner Gesellschaften. Soziologie, 47, 482-489.

Ettrich, F. (1991). Von der Erfolgswissenschaft zur Krisenwissenschaft. Die gesellschaftlichen Transformationsprozesse in Osteuropa und Ostdeutschland als Herausforderung der deutschen Soziologie. In H. Meyer (Hrsg.), Soziologen-Tag Leipzig 1991 (S. 122-145). Berlin: Akademie Verlag.

Federici, S. (2015). Caliban und die Hexe. Frauen, der Körper und die ursprüngliche Akkumulation. Wien: Mandelbaum.

Gerhardt, V. (2019). Die normative Wende im Anthropozän. In P.J. Crutzen, Das Anthropozän. Schlüsseltexte des Nobelpreisträgers für das neue Erdzeitalter (S. 79-89). München: oekom.

Giddens, A. (1995). Konsequenzen der Moderne. Frankfurt a.M.: Suhrkamp.

Haag, H., Leonhard, N., \& Heß, P. (Hrsg.). (2017). Volkseigenes Erinnern. Die DDR im sozialen Gedächtnis. Wiesbaden: Springer VS.

Hillebrand, M. (2014). Ostdeutsche Selbstwahrnehmungen und Identitätskonstruktionen im Generationenvergleich. Frankfurt a.M.: Internationaler Verlag der Wissenschaften.

IPCC (2018). Special Report. Global Warming of $1,5^{\circ}$ C. Bonn: UN Climate Change.

Lessenich, S. (2020a). Doppelmoral hält besser: Die Politik mit der Solidarität in der Externalisierungsgesellschaft. Berliner Journal für Soziologie, 30, 113-130.

Lessenich, S. (2020b). Soziologie - Corona - Kritik. Berliner Journal für Soziologie, 30, 215-230.

Löw, M., \& Knoblauch, H. (2021). Raumfiguren, Raumkulturen und die Refiguration von Räumen. In M. Löw, V. Saymann, J. Schwerer \& H. Wolf (Hrsg.), Am Ende der Globalisierung. Über die Refiguration von Räumen (S. 25-57). Bielefeld: transcript.

Mau, S. (2019). Lütten Klein. Leben in der ostdeutschen Transformationsgesellschaft. Berlin: Suhrkamp.

Mau, S., Lux, T., \& Gülzau, F. (2020). Die drei Arenen der neuen Ungleichheitskonflikte. Eine sozialstrukturelle Positionsbestimmung der Einstellungen zu Umverteilung, Migration und sexueller Diversität. Berliner Journal für Soziologie, 30. 317-346. 
Menzel, Ulrich (2021). Corona und die gefesselte Globalisierung. Berliner Journal für Soziologie. https:// doi.org/10.1007/s11609-021-00437-7.

Moore, J. W. (2015). Capitalism in the web of life. Ecology and the accumulation of capital. London: Verso.

Norgaard, K.M. (2017). The sociological imagination in a time of climate change. Global and Planetary Change, 163, 171-176.

Polanyi, K. (1995) [1944]. The Great Transformation. Politische und ökonomische Ursprünge von Gesellschaften und Wirtschaftssystemen. Frankfurt a.M.: Suhrkamp.

Rogelj, J., Forster, P. M., Kriegler, E., Smith, C. J., \& Séférian, R. (2019). Estimating and tracking the remaining carbon budget for stringent climate targets. Nature, 571, 335-342.

Rosa, H. (2020). Pfadabhängigkeit, Bifurkationspunkte und die Rolle der Soziologie. Ein soziologischer Deutungsversuch der Corona-Krise. Berliner Journal für Soziologie, 30, 191-213.

Seyd, B. (2020). Corona - Krise - Kritik. Eine Kontroverse im Berliner Journal für Soziologie. Berliner Journal für Soziologie, 30, 157-163.

Ther, P. (2014). Die neue Ordnung auf dem alten Kontinent. Eine Geschichte des neoliberalen Europa. Berlin: Suhrkamp.

UNEP (2019). Emissions Gap Report 2019. Nairobi.

Waynwright, J., \& Mann, G. (2018). Climate Leviathan: A political theory of our planetary future. London: Verso.

Weizsäcker, E. U. v. (2020). Eine spannende Reise zur Nachhaltigkeit. Naturkapitalismus und die neue Aufklärung. In B. Görgen \& B. Wendt (Hrsg.), Sozial-ökologische Utopien. Diesseits oder jenseits von Wachstum und Kapitalismus? (S. 81-95). München: oekom.

Zapf, Wolfgang (1994): Transformation in der ehemaligen DDR und die soziologische Theorie der Modernisierung. Berliner Journal für Soziologie, 4, 295-305. 